\title{
IDEMPOTENT-SEPARATING EXTENSIONS OF REGULAR SEMIGROUPS WITH ABELIAN KERNEL
}

\author{
M. LOGANATHAN
}

(Received 10 March 1980)

Communicated by T. E. Hall

\begin{abstract}
Let $S$ be a regular semigroup and $D(S)$ its associated category as defined in Loganathan (1981). We introduce in this paper the notion of an extension of a $D(S)$-module $A$ by $S$ and show that the set $\operatorname{Ext}(S, A)$ of equivalence classes of extensions of $A$ by $S$ forms an abelian group under a Baer sum. We also study the functorial properties $\operatorname{Ext}(S, A)$.
\end{abstract}

1980 Mathematics subject classification (Amer. Math. Soc.): 20 M 50.

\section{Introduction}

Let $S$ be a regular semigroup and $D(S)$ its associated category (see Section 2 for the definition of $D(S)$ ). By a $D(S)$-module we mean a functor $A: D(S) \rightarrow A b$ from $D(S)$ to the category of abelian groups and by a $D(S)$-homomorphism a natural transformation between such functors.

Let $\pi: T \rightarrow S$ be an idempotent-separating homomorphism from a regular semigroup $T$ onto $S$. If the kernel of $\pi$ is abelian then the kernel can be viewed as a $D(S)$-module $\operatorname{Ker} \pi: D(S) \rightarrow A b$. This suggests the following definition: An extension of a $D(S)$-module $A$ by $S$ is a triple $(T, \pi, i)$ consisting of a regular semigroup $T$, an idempotent-separating surjective homomorphism of regular semigroups $\pi: T \rightarrow S$ with abelian kernel and an isomorphism $i: A \rightarrow \operatorname{Ker} \pi$ of $D(S)$-modules. Let $\operatorname{Ext}(S, A)$ denote the set of equivalence classes of extensions of $A$ by $S$. We define an addition in $\operatorname{Ext}(S, A)$ and show that it makes $\operatorname{Ext}(S, A)$ an abelian group. We also study the functorial properties of $\operatorname{Ext}(S, A)$.

(1) Copyright Australian Mathematical Society 1982 
The cohomological interpretation of $\operatorname{Ext}(S, A)$ will be given in another paper. Here we content ourselves with the following remarks. (For details see Loganathan (1981).) When $G$ is a group the category $D(G)$ reduces to the group $G$ itself and in this case our results are well known and classical. When $S$ is an inverse semigroup then the concept of the $D(S)$-module is equivalent to that of the $S$-module as defined by Lausch (1975). Consequently, in the case of semi-lattices of groups, our results turn out to be equivalent to those obtained by Sribala (1977). For regular semigroups the category $D(S)$ is equivalent to the category $\mathscr{D}(S)$ introduced by Leech (1975). In particular, the type of extensions considered in this paper is a special case of the $\mathcal{H}$-coextensions studied by Leech.

\section{Preliminaries}

Let $S$ be a regular semigroup and $E(S)$ its set of idempotents. For $x \in S$, we denote by

$$
V(x)=\left\{x^{\prime} \in S \mid x x^{\prime} x=x \text { and } x^{\prime} x x^{\prime}=x^{\prime}\right\}
$$

the set of inverses of $x$ in $S$. If $x^{\prime} \in V(x)$ then $\left(x, x^{\prime}\right)$ will be called a regular pair in $S$. For $e, f \in E(S)$, we denote by

$$
S(e, f)=\{h \in E(S) \mid h e=h=f h \text { and } e h f=e f\}
$$

the sandwich set of $e$ and $f$ (Nambooripad (1979)).

If $\rho$ is a congruence on $S$ then the kernel of $\rho$ is the set of $\rho$-classes which contain idempotents of $S$. By the kernel of a homomorphism we mean the kernel of its associated congruence. If $\rho$ is idempotent-separating then, since $\rho \subseteq \mathcal{H}$, for each $e \in E(S)$ the $\rho$-class $N_{e}$ containing the idempotent $e$ is a subgroup of $S$. The kernel of an idempotent-separating congruence $\rho$ is called abelian if the groups $N_{e}$ are abelian.

A set $N=\left\{N_{e} \mid e \in E(S)\right\}$, of subgroups of $S$, is called a group kernel normal system if the following hold

(i) $e \in N_{e}$ for each $e \in E(S)$;

(ii) $a f=f a$ for each $a \in N_{e}$ and $f \leqslant e, f \in E(S)$;

(iii) $x^{\prime} N_{e} x \subseteq N_{x^{\prime} x}$ for each regular pair $\left(x, x^{\prime}\right)$ of $S$ with $x x^{\prime} \leqslant e$.

Let $N=\left\{N_{e} \mid e \in E(S)\right\}$ be a group kernel normal system of $S$. We denote by $\rho_{N}$ the relation on $S$ defined by

$$
\begin{aligned}
& \left\{(x, y) \in S \times S \mid \text { for some } x^{\prime} \in V(x) \text { and } y^{\prime} \in V(y),\right. \\
& \left.x x^{\prime}=y y^{\prime}, x^{\prime} x=y^{\prime} y \text { and } y^{\prime} x \in N_{x^{\prime} x}\right\} .
\end{aligned}
$$


By Lallement (1967), Theorem 3.11, $\rho_{N}$ is an idempotent-separating congruence on $S$ with $N$ as its kernel.

Next we recall the definition of the category $D(S)$ introduced in Loganathan (1981).

Let $S$ be a regular semigroup. Let $C(S)$ denote the category whose objects are the idempotents of $S$, where a morphism from the idempotent $e$ to the idempotent $f$ is a triple $\left(e, x, x^{\prime}\right)$ with $\left(x, x^{\prime}\right)$ a regular pair in $S$ satisfying $x x^{\prime}<e$, $x^{\prime} x=f$. Composition is defined by $\left(e, x, x^{\prime}\right)\left(x^{\prime} x, y, y^{\prime}\right)=\left(e, x y, y^{\prime} x^{\prime}\right)$. Composition is clearly associative and $(e, e, e)$ is the identity morphism at the object e.

On $C(S)$ define a relation $\rho$ as follows. If $\left(e, x, x^{\prime}\right),\left(e, y, y^{\prime}\right): e \rightarrow f$ are morphisms from $e$ to $f$ then

$$
\left(e, x, x^{\prime}\right) \rho\left(e, y, y^{\prime}\right) \text { if and only if } x=y \text { or } x^{\prime}=y^{\prime} \text {. }
$$

The relation $\rho$ is clearly reflexive and symmetric and compatible with composition. Hence $\rho^{t}$, the transitive closure of $\rho$, is a congruence on $C(S)$. We write $D(S)$ for the quotient category $C(S) / \rho^{t}$. If $\left(e, x, x^{\prime}\right)$ is a morphism of $C(S)$, we shall write $\left[e, x, x^{\prime}\right]$ for the image of $\left(e, x, x^{\prime}\right)$ in $D(S)$. Let $\theta: S^{\prime} \rightarrow S$ be a homomorphism of regular semigroups. Then the maps $e \mapsto e \theta,\left[e, x, x^{\prime}\right] \mapsto$ $\left[e \theta, x \theta, x^{\prime} \theta\right.$ ], define a functor $D(\theta): D\left(S^{\prime}\right) \rightarrow D(S)$.

Definition 2.1. A map $x \mapsto x^{*}: S \rightarrow S$ is called an inverse map if (i) $x^{*} \in$ $V(x)$ for each $x \in S$; (ii) $x^{*} \in H_{e}$ if $x \in H_{e}$. In particular, if $e \in E(S)$ then $e^{*}=e$.

Let $S$ be a regular semigroup with an inverse map $x \mapsto x^{*}$. For $x, y \in S$, we denote by $K_{x y}$ the morphism

$$
\left[y^{*} y, y^{*} y(x y)^{*} x y,(x y)^{*} x y\right]: y^{*} y \rightarrow(x y)^{*} x y
$$

and by $J_{x y}$ the morphism

$$
\left[x^{*} x, x^{*} x y,(x y)^{*} x h\right]: x^{*} x \rightarrow(x y)^{*} x y,
$$

where $h$ is any element of $S\left(x^{*} x, y y^{*}\right)$. Clearly $J_{x_{y}}$ does not depend on the choice of $h$ and hence it is well-defined.

LEMMA 2.2. For $x, y, z \in S$, we have

(i) $J_{x, y} J_{x y, z}=J_{x, y z}$;

(ii) $K_{y, z} K_{x, y z}=K_{x y, z}$;

(iii) $K_{x, y} J_{x y, z}=J_{y, z} K_{x, y z}$. 
Proof. (i) and (ii) are easy to verify and (iii) also follows by observing that

$$
\begin{aligned}
K_{x, y} J_{x y, z} & =\left[y^{*} y, y^{*} y(x y)^{*} x y z,(x y z)^{*} x y h\right] \\
& =\left[y^{*} y, y^{*} y z(x y z)^{*} x y z,(x y z)^{*} x y h\right] \\
& =\left[y^{*} y, y^{*} y z(x y z)^{*} x y z,(x y z)^{*} x y k\right] \\
& =J_{y, z} K_{x, y z},
\end{aligned}
$$

where $h, k$ are any elements of $S\left((x y)^{*} x y, z z^{*}\right)$ and $S\left(y^{*} y, z z^{*}\right)$ respectively.

\section{The group $\operatorname{Ext}(S, A)$}

Let $T, S$ be regular semigroups and let $\pi: T \rightarrow S$ be an idempotent-separating homomorphism from $T$ onto $S$. For $e \in E(S)$, we denote by (Ker $\pi)_{e}$ the group $\{t \in T \mid t \pi=e\}$. For each morphism $\left[h, t, t^{\prime}\right]: h \mapsto k$ of $D(T)$ we define

$$
K\left(\left[h, t, t^{\prime}\right]\right):(\operatorname{Ker} \pi)_{h \pi} \rightarrow(\operatorname{Ker} \pi)_{k \pi}
$$

by $a K\left(\left[h, t, t^{\prime}\right]\right)=t^{\prime} a t, a \in(\operatorname{Ker} \pi)_{h \pi}$. It is easy to see that $K\left(\left[h, t, t^{\prime}\right]\right)$ is welldefined and that it is a homomorphism of groups. Therefore the maps

$$
h \mapsto(\operatorname{Ker} \pi)_{h \pi}, \quad\left[h, t, t^{\prime}\right] \mapsto K\left(\left[h, t, t^{\prime}\right]\right)
$$

define a functor $K$ from $D(T)$ to the category of groups.

Suppose now that the kernel of $\pi$ is abelian, that is, the groups $(\operatorname{Ker} \pi)_{e}$, $e \in E(S)$, are abelian. Then $K: D(T) \rightarrow A b$ induces a $D(S)$-module Ker $\pi$ : $D(S) \rightarrow A b$ such that the diagram

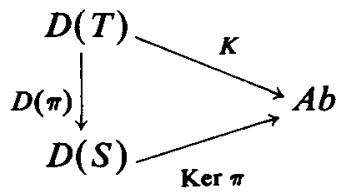

is commutative. By assigning to each regular pair $\left(x, x^{\prime}\right)$ in $S$ a regular pair $\left(\left(x, x^{\prime}\right) j_{1},\left(x, x^{\prime}\right) j_{2}\right)$ in $T$ such that $\left(\left(x, x^{\prime}\right) j_{1} \pi,\left(x, x^{\prime}\right) j_{2} \pi\right)=\left(x, x^{\prime}\right)$, we see that Ker $\pi$ can be described, more explicitly, as the $D(S)$-module which associates to each object $e$ of $D(S)$ the group $(\operatorname{Ker} \pi)_{e}$ and to each morphism [e, $\left.x, x^{\prime}\right]: e \rightarrow f$ the homomorphism Ker $\pi\left(\left[e, x, x^{\prime}\right]\right):(\operatorname{Ker} \pi)_{e} \rightarrow(\operatorname{Ker} \pi)_{f}$, given by

$$
\text { (a) } \operatorname{Ker} \pi\left(\left[e, x, x^{\prime}\right]\right)=\left(x, x^{\prime}\right) j_{2} a\left(x, x^{\prime}\right) j_{1}, \quad a \in(\operatorname{Ker} \pi)_{e} \text {. }
$$

Motivated by the above observation we introduce the following

Definition 3.1. Let $S$ be a regular semigroup and $A$ a $D(S)$-module. An extension of $A$ by $S$ is a triple $E=(T, \pi, i)$ consisting of a regular semigroup $T$, an idempotent-separating surjective homomorphism $\pi: T \rightarrow S$ such that the kernel of $\pi$ is abelian, and an isomorphism of $D(S)$-modules $i: A \rightarrow \operatorname{Ker} \pi$. 
Consider the commutative diagram

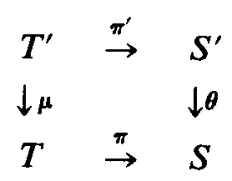

of homomorphisms of regular semigroups such that $\pi^{\prime}$ and $\pi$ are idempotentseparating surjective homomorphisms with abelian kernel. Let $D(\theta)(\operatorname{Ker} \pi)$ be the composite

$$
D\left(S^{\prime}\right) \stackrel{D(\theta)}{\rightarrow} D(S) \stackrel{\operatorname{Ker} \pi}{\rightarrow} A b .
$$

Then $\mu$ induces a homomorphism $\phi_{\mu}: \operatorname{Ker} \pi^{\prime} \rightarrow D(\theta)(\operatorname{Ker} \pi)$ of $D\left(S^{\prime}\right)$-modules such that

$$
\left.\phi_{\mu}\right|_{\left(\operatorname{Ker} \pi^{\prime}\right)_{e}}=\left.\mu\right|_{\left(\operatorname{Ker} \pi^{\prime}\right)_{e}}:\left(\operatorname{Ker} \pi^{\prime}\right)_{e} \rightarrow D(\theta)(\operatorname{Ker} \pi)_{e}=(\operatorname{Ker} \pi)_{e \theta} .
$$

Let $E^{\prime}=\left(T^{\prime}, \pi^{\prime}, i^{\prime}\right)$ be an extension of $A^{\prime}$ by $S^{\prime}$ and $E=(T, \pi, i)$ an extension of $A$ by $S$. A morphism $\Gamma: E^{\prime} \rightarrow E$ of extensions is a triple $\Gamma=(\phi, \mu, \theta)$ consisting of homomorphisms $\mu: T^{\prime} \rightarrow T, \theta: S^{\prime} \rightarrow S$ of regular semigroups and a homomorphism $\phi: A^{\prime} \rightarrow D(\theta) A$ of $D\left(S^{\prime}\right)$-modules, such that: $\mu \pi=\pi^{\prime} \theta$ and $\phi D(\theta) i=i^{\prime} \phi_{\mu}$, where

$$
D(\theta) i: D(\theta) A \rightarrow D(\theta)(\operatorname{Ker} \pi)
$$

is the homomorphism of $D\left(S^{\prime}\right)$-modules induced by $i: A \rightarrow \operatorname{Ker} \pi$. We say two extensions $E^{\prime}=\left(T^{\prime}, \pi^{\prime}, i^{\prime}\right), E=(T, \pi, i)$ of $A$ by $S$ are equivalent if there exists a homomorphism (necessarily an isomorphism) $\mu: T^{\prime} \rightarrow T$ such that $\left(\operatorname{Id}_{A}, \mu, \mathrm{Id}_{S}\right): E^{\prime} \rightarrow E$ is a morphism of extensions. We call $\mu$ an equivalence of extensions. We denote by $\operatorname{Ext}(S, A)$ the equivalence classes of extensions of $A$ by $S$ and by $[E]$ the equivalence class containing the extension $E=(T, \pi, i)$.

Definition 3.2. Let $S$ be a regular semigroup and $A$ a $D(S)$-module. The semi-direct product of $S$ and $A$ with respect to an inverse map $x \mapsto x^{*}: S \rightarrow S$ is the regular semigroup

$$
S \times A=\left\{(x, a) \mid x \in S, a \in A_{x^{*} x}\right\}
$$

with the multiplication given by

$$
(x, a)(y, b)=\left(x y, a A\left(J_{x, y}\right)+b A\left(K_{x y}\right)\right) .
$$

Associativity of the multiplication follows from Lemma 2.2. The set $E(S \times A)$ of idempotents of $S \times A$ is $\left\{\left(e, 0_{e}\right) \in S \times A \mid e \in E(S)\right\}$ and if $(x, a) \in S \times A$ then

$$
V\left((x, a)=\left\{\left(y,(-a) A\left(\left[x^{*} x, x^{*} x y, y^{*} y x\right]\right)\right) \in S \times A \mid y \in V(x)\right\} .\right.
$$


Suppose now that $S \times A$ is the semi-direct product of $S$ and $A$ with respect to an inverse map $x \mapsto x^{*}$. Then the projection $\pi_{0}: S \times A \rightarrow S$, defined by $(x, a) \pi_{0}$ $=x$, and the isomorphism $i_{0}: A \rightarrow \operatorname{Ker} \pi_{0}$, given by $(a) i_{0}=(e, a), a \in A_{e}$, $e \in E(S)=\mathrm{Ob}(D(S))$, of $D(S)$-modules define an extension, denoted $E_{0}=(S$ $\left.\times A, \pi_{0}, i_{0}\right)$, of $A$ by $S$. Also, there is a homomorphism $\nu_{0}: S \rightarrow S \times A$, given by $(x) \nu_{0}=\left(x, 0_{x^{*} x}\right)$, which satisfies $\nu_{0} \pi_{0}=I_{S}$.

We call an extension $E=(T, \pi, i)$ of $A$ by $S$ split if there exists a homomorphism $\nu: S \rightarrow T$ such that $\nu \pi=\operatorname{Id}_{S}$; the homomorphism $\nu$ is then called a splitting. For example $E_{0}$ is a split extension with a splitting $\nu_{0}$.

If $E=(T, \pi, i)$ is a split extension of $A$ by $S$ with a splitting $\nu$ then the map $\mu$ : $S \times A \rightarrow T$, given by $(x, a) \mu=(x v)(a i)$, is a homomorphism of regular semigroups and it is an equivalence of extensions. Conversely, if an extension $E$ is equivalent to $E_{0}$ then, obviously, it is a split extension. In other words, an extension $E$ of $A$ by $S$ is split if and only if it is equivalent to $E_{0}$. In particular, the equivalence class determined by $E_{0}$ does not depend on the particular choice of the inverse map $x \mapsto x^{*}$ used to define the multiplication in $S \times A$.

Let $E_{r}=\left(T_{r}, \pi_{r}, i_{r}\right), r=1,2$, be extensions of $A$ by $S$. Consider the regular subsemigroup

$$
T=\left\{\left(t_{1}, t_{2}\right) \in T_{1} \times T_{2} \mid t_{1} \pi_{1}=t_{2} \pi_{2}\right\}
$$

of $T_{1} \times T_{2}$. Since $\pi_{1}$ and $\pi_{2}$ are idempotent-separating homomorphisms, the set $N=\left\{N_{e} \mid e \in E(S)\right\}$, where $N_{e}=\left\{\left((a) i_{1},(-a) i_{2}\right) \mid a \in A_{e}\right\}$, is a group kernel normal system of $T$. Write $T_{1}+T_{2}=T / \rho_{N}$ and denote the element of $T_{1}+T_{2}$ containing $\left(t_{1}, t_{2}\right)$ by $\overline{\left(t_{1}, t_{2}\right)}$. It is easy to see that the map $\pi: T_{1}+T_{2} \rightarrow S$, given by $\overline{\left(t_{1}, t_{2}\right)} \pi=t_{1} \pi_{1}\left(=t_{2} \pi_{2}\right)$, is an idempotent-separating homomorphism from $T_{1}+T_{2}$ onto $S$. Also, it is easy to see that the map $i: A \rightarrow \operatorname{Ker} \pi$, defined by (a) $i=\frac{2}{\left((a) i_{1},(0) i_{2}\right)}, a \in A_{e}, e \in E(S)$, is an isomorphism of $D(S)$-modules. Thus we obtain an extension, denoted $E_{1}+E_{2}=\left(T_{1}+T_{2}, \pi, i\right)$, of $A$ by $S$. We call $E_{1}+E_{2}$ the Baer sum of the extensions $E_{1}$ and $E_{2}$.

Throrem 3.3. $\operatorname{Ext}(S, A)$ is an abelian group under the operation $\left[E_{1}\right]+\left[E_{2}\right]=$ $\left[E_{1}+E_{2}\right]$. The zero element in the abelian group $\operatorname{Ext}(S, A)$ is the equivalence class $\left[E_{0}\right]$ determined by the split extensions.

PROOF. Commutativity of + . Let $E_{r}=\left(T_{r}, \pi_{r}, i_{r}\right), r=1,2$, be extensions of $A$ by $S$. Then the map $\mu$ : $T_{1}+T_{2} \rightarrow T_{2}+T_{1}$, given by $\overline{\left(t_{1}, t_{2}\right)} \mu=\overline{\left(t_{2}, t_{1}\right)}$, is easily seen to be an equivalence of extensions so that $\left[E_{1}\right]+\left[E_{2}\right]=\left[E_{1}+E_{2}\right]=\left[E_{2}\right]$ $+\left[E_{1}\right]$.

Associativity of + . Let $E_{r}=\left(T_{r}, \pi_{r}, i_{r}\right), r=1,2,3$, be extensions of $A$ by $S$. We have to show that $\left(E_{1}+E_{2}\right)+E_{3}$ is equivalent to $E_{1}+\left(E_{2}+E_{3}\right)$. This 
follows by noting that the map $\mu:\left(T_{1}+T_{2}\right)+T_{3} \rightarrow T_{1}+\left(T_{2}+T_{3}\right)$, defined by

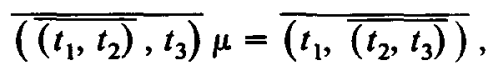

is an equivalence of extensions.

Identity for + . Let $E=(T, \pi, i)$ be an extension of $A$ by $S$. Then the map $\mu$ : $T \rightarrow T+(S \times A)$, given by $t \mu=\overline{\left(t,\left(t \pi, 0_{(t \pi) * t \pi}\right)\right)}$, is an equivalence of extensions between $E$ and $E+E_{0}$. Hence $[E]+\left[E_{0}\right]=\left[E+E_{0}\right]=[E]=\left[E_{0}\right]+$ [E].

Inverse (relative to + ). Let $E=(T, \pi, i)$ be an extension of $A$ by $S$. Denote by $-E=(T, \pi,-i)$ the extension obtained from $E$ by defining $a(-i)=(-a) i$, $a \in A$. We claim that $[-E]$ is the inverse of $[E]$. It clearly suffices to show that the extension $E+(-E)$ is split. Choose a map $j: S \rightarrow T$ such that $j \pi=\operatorname{Id}_{s}$. Define $\nu: S \rightarrow T+T$ by $(x) \nu=\overline{(x j, x j)}$. It follows from the definition of $E+(-E)$ that the map $\nu$ is a homomorphism. Obviously $\nu \pi=\mathrm{Id}_{s}$. Hence $E+(-E)$ is a split extension. This completes the proof of the theorem

Next we will study the functorial properties of $\operatorname{Ext}(S, A)$.

Proposition 3.4. Let $E=(T, \pi, i)$ be an extension of $A$ by $S$ and let $\phi: A \rightarrow B$ be a homomorphism of $D(S)$-modules. Then there is an extension $\phi E=\left(U, \pi^{\prime}, i^{\prime}\right)$ of $B$ by $S$ and a homomorphism $\mu: T \rightarrow U$ such that $\left(\phi, \mu, \mathrm{Id}_{S}\right): E \rightarrow \phi E$ is a morphism of extensions. The pair $(\phi E, \mu)$ is unique upto an equivalence.

Proof. Let $T \times B^{\prime}$ be the semi-direct product of $T$ and $B^{\prime}$ with respect to an inverse map $t \mapsto t^{*}: T \rightarrow T$, where $B^{\prime}$ denote the composite $D(T) \stackrel{D(\pi)}{\rightarrow} D(S) \stackrel{B}{\rightarrow} A b$. For $\left(e, 0_{e}\right) \in E\left(T \times B^{\prime}\right)$, let

$$
N_{\left(e, 0_{e}\right)}=\left\{((a) i,(-a) \phi) \in T \times B^{\prime} \mid a \in A_{e \pi}\right\} .
$$

In view of the bijection $\left(e, 0_{e}\right) \leftrightarrow e$ between $E\left(T \times B^{\prime}\right)$ and $E(T)$, we denote the group $N_{\left(e, 0_{e}\right)}$ simply by $N_{e}$. We shall prove that $N=\left\{N_{e} \mid e \in E(T)\right\}$ is a group kernel normal system of $T \times B^{\prime}$.

(i) Clearly $\left(e, 0_{e}\right) \in N_{e}$, for each idempotent $\left(e, 0_{e}\right)$ of $T \times B^{\prime}$.

(ii) Suppose that $((a) i,(-a) \phi) \in N_{e}$ and let $\left(f, 0_{f}\right) \leqslant\left(e, 0_{e}\right)$. Then $f<e$ in $T$ so that $(a i) f=f(a i)$ and, by Definition 2.1(ii), $((a i) f)^{*}=((-a) i) f$. It follows that

$$
((a) i,(-a) \phi)\left(f, 0_{f}\right)=\left(f, 0_{f}\right)((a) i,(-a) \phi) .
$$

(iii) Suppose that $((a) i,(-a) \phi) \in N_{e}$ and let

$$
\left((t, b),\left(u,(-b) B^{\prime}\left(\left[t^{*} t, t^{*} t u, u^{*} u t\right]\right)\right)\right.
$$


be a regular pair in $T \times B^{\prime}$ such that

$$
(t, b)\left(u,(-b) B^{\prime}\left(\left[t^{*} t, t^{*} t u, u^{*} u t\right]\right)\right)=\left(t u, 0_{t u}\right)<\left(e, 0_{e}\right) .
$$

Since $(u(a) i)^{*} u(a) i \mathcal{L} u^{*} u £ t u, t u \in S\left((u(a) i)^{*} u(a) i, t t^{*}\right)$. Therefore,

$$
\begin{gathered}
\left(u,(-b) B^{\prime}\left(\left[t^{*} t, t^{*} t u, u^{*} u t\right]\right)\right)((a) i,(-a) \phi)(t, b) \\
=\left(u(a i) t,(-b) B^{\prime}\left(\left[t^{*} t, t^{*}(a i) t, u(u(a i))^{*} u t\right]\right)\right. \\
\left.\quad+(-a) \phi B^{\prime}([e, t, u])+b B^{\prime}\left(\left[t^{*} t, t^{*} t, u t\right]\right)\right) \\
=\left(u(a i) t,(-a) \phi B^{\prime}([e, t, u])\right),
\end{gathered}
$$

since

$$
B^{\prime}\left(\left[t^{*} t, t^{*}(a i) t, u(u(a i))^{*} u t\right]\right)=B^{\prime}\left(\left[t^{*} t, t^{*} t, u t\right]\right) .
$$

Now, by putting $a^{\prime}=a A([e \pi, t \pi, u \pi])$, we see that

$$
\left(u(a i) t,(-a) \phi B^{\prime}([e, t, u])\right)=\left(\left(a^{\prime}\right) i,\left(-a^{\prime}\right) \phi\right) \in N_{u t} .
$$

Hence $N=\left\{N_{e} \mid e \in E(T)\right\}$ is a group kernel normal system of $T \times B^{\prime}$.

Write $U=T \times B^{\prime} / \rho_{N}$. The composite $T \times B^{\prime} \stackrel{\pi_{0}}{\rightarrow} T \stackrel{\pi}{\rightarrow} S$ induces a surjective homomorphism $\pi^{\prime}: U \rightarrow S$, which is idempotent-separating. If we define $i^{\prime}$ : $B \rightarrow \operatorname{Ker} \pi^{\prime}$ by $(b) i^{\prime}=(e, b) \rho_{N}, b \in B_{e}^{\prime}$, then, clearly, $i^{\prime}$ is a monomorphism. It is also an epimorphism. For, if $\left((t, b) \rho_{N}\right) \pi^{\prime}=\left(\left(e, 0_{e}\right) \rho_{N}\right) \pi^{\prime}$, for some $\left(e, 0_{e}\right) \rho_{N} \in$ $E(U)$, then $t \pi=e \pi$ so that we can find an element $a \in A_{e \pi}$ such that $(a) i=t$. Hence $(t, b) \rho_{N}=(e,(a) \phi-b) \rho_{N}=((a) \phi-b) i^{\prime}$. Therefore $i^{\prime}$ is an isomorphism of $D(S)$-modules. Consequently, $\left(U, \pi^{\prime}, i^{\prime}\right)$ is an extension of $B$ by $S$ which we denote by $\phi E$. Finally, we define $\mu: T \rightarrow U$ by $(t) \mu=\left(t, 0_{t^{*}}\right) \rho_{N}$. It is easily seen that $\left(\phi, \mu, \mathrm{Id}_{s}\right): E \rightarrow \phi E$ is a morphism of extensions.

Suppose that $\left(\left(V, \pi^{\prime \prime}, i^{\prime \prime}\right), \mu^{\prime}\right)$ is any other such pair. Then the map $\mu^{\prime \prime}$ : $T \times B^{\prime} \rightarrow V$, defined by $(t, a) \mu^{\prime \prime}=(t) \mu^{\prime}(a) i^{\prime \prime}$, is easily seen to be a homomorphism of regular semigroups. If $((a) i,(-a) \phi) \in N_{e}$ then $((a) i,(-a) \phi) \mu^{\prime \prime}=$ $(a i) \mu^{\prime}((-a) \phi) i^{\prime \prime}=(a \phi) i^{\prime \prime}((-a) \phi) i^{\prime \prime}=0$. Therefore $\mu^{\prime \prime}$ induces a homomorphism $\bar{\mu}: U \rightarrow V$. Clearly $\bar{\mu}$ is an equivalence of extensions and satisfies the equation $\mu \bar{\mu}=\mu^{\prime}$. This completes the proof of the proposition.

Proposition 3.5. Let $E=(T, \pi, i)$ be an extension of $A$ by $S$ and let $\theta: S^{\prime} \rightarrow S$ be a homomorphism of regular semigroups. Let $D(\theta) A$ denote the composite $D\left(S^{\prime}\right) \stackrel{D(\theta)}{\rightarrow} D(S) \stackrel{A}{\rightarrow} A b$. Then there is an extension $E \theta=\left(T^{\prime}, \pi^{\prime}, i^{\prime}\right)$ of $D(\theta) A$ by $S^{\prime}$ and a homomorphism $\mu: T^{\prime} \rightarrow T$ of regular semigroups such that $\left(\operatorname{Id}_{D(\theta) A}, \mu, \theta\right)$ : $E \theta \rightarrow E$ is a morphism of extensions. The pair $(E \theta, \mu)$ is unique up to an equivalence. 
Proof. Let $T^{\prime}=\left\{(x, t) \in S^{\prime} \times T \mid x \theta=t \pi\right\}$ be the regular subsemigroup of $S^{\prime} \times T$. The projection $\pi^{\prime}: T^{\prime} \rightarrow S^{\prime}$, defined by $(x, t) \pi^{\prime}=x$, and the homomorphism $i^{\prime}: D(\theta) A \rightarrow \operatorname{Ker} \pi^{\prime}$ of $D\left(S^{\prime}\right)$-modules, given by $(a) i^{\prime}=(e,(a) i), e \in$ $E\left(S^{\prime}\right), a \in(D(\theta) A)_{e}=A_{e \theta}$, define an extension $E \theta=\left(T^{\prime}, \pi^{\prime}, i^{\prime}\right)$. If we define $\mu$ : $T^{\prime} \rightarrow T$ by $(x, t) \mu=t,(x, t) \in T^{\prime}$, then, clearly $\left(\operatorname{Id}_{D(\theta) A}, \mu, \theta\right): E \theta \rightarrow E$ is a morphism of extensions.

Suppose that $\left(E^{\prime}=\left(T^{\prime \prime}, \pi^{\prime \prime}, i^{\prime \prime}\right), \mu^{\prime \prime}\right)$ is any other such pair. Then the homomorphism $\mu^{\prime}: T^{\prime \prime} \rightarrow T^{\prime}$, given by $(t) \mu^{\prime}=\left(t \pi^{\prime \prime}, t \mu^{\prime \prime}\right)$, is an equivalence of extensions and satisfies the equation $\mu^{\prime} \mu=\mu^{\prime \prime}$. Hence $(E \theta, \mu)$ is unique up to an equivalence.

The next result is immediate from Propositions 3.4 and 3.5.

Proposition 3.6. Let $\theta: S^{\prime} \rightarrow S$ be a homomorphism of regular semigroups and let $\phi: A \rightarrow B$ be a homomorphism of $D(S)$-modules. If $E=(T, \pi, i)$ is an extension of $A$ by $S$ then the extension $(D(\theta) \phi)(E \theta)$ is equivalent to the extension $(\phi E) \theta$. Here $D(\theta) \phi$ denote the homomorphism $D(\theta) A \rightarrow D(\theta) B$ of $D\left(S^{\prime}\right)$-modules.

We shall write $\mathcal{C}$ for the following category: an object of $\mathcal{C}$ is a pair $(S, A)$ with $S$ a regular semigroup and $A$ a $D(S)$-module; a morphism $(\theta, \phi):(S, A) \rightarrow$ $(T, B)$ in $\mathcal{C}$ consists of a homomorphism $\theta: S \rightarrow T$ of regular semigroups and a homomorphism $\phi: D(\theta) B \rightarrow A$ of $D(S)$-modules.

THEOREM 3.7. The mapping which associates to each object $(S, A)$ of $\mathcal{C}$, the abelian group $\operatorname{Ext}(S, A)$ and, to each morphism $(\theta, \phi):(S, A) \rightarrow(T, B)$, the homomorphism $\operatorname{Ext}(\theta, \phi): \operatorname{Ext}(T, B) \rightarrow \operatorname{Ext}(S, A)$ given by the composite $\operatorname{Ext}(T, B) \rightarrow \operatorname{Ext}(S, D(\theta) B) \rightarrow \operatorname{Ext}(S, A)$ is a contravariant functor from $\mathcal{C}$ to the category of abelian groups.

Proof. If $\theta: S \rightarrow T$ is a homomorphism of regular semigroups and $B$ is a $D(T)$-module then, by Proposition 3.5, the map $[E] \mapsto[E \theta]: \operatorname{Ext}(T, B) \rightarrow$ $\operatorname{Ext}(S, D(\theta) B)$ is a homomorphism of abelian groups. Similarly, if $S$ is a regular semigroup, then by Proposition 3.4, $\operatorname{Ext}(S,-)$ is a functor from the category of $D(S)$-modules to the category of abelian groups. In particular, if $\phi: A \rightarrow A^{\prime}$ is a homomorphism of $D(S)$-modules then $[E] \mapsto[\phi E]: \operatorname{Ext}(S, A) \rightarrow \operatorname{Ext}\left(S, A^{\prime}\right)$ is a homomorphism of abelian groups. Therefore if $(\theta, \phi):(S, A) \rightarrow(T, B)$ is a morphism of $\mathcal{C}$ then $\operatorname{Ext}(\theta, \phi)$ is a homomorphism of abelian groups from $\operatorname{Ext}(T, B)$ to $\operatorname{Ext}(S, A)$. It remains to show that $\operatorname{Ext}(-,-)$ is contravariant. Let 
$(\theta, \phi):(S, A) \rightarrow(T, B)$ and $(\mu, \psi):(T, B) \rightarrow(U, C)$ be morphisms of $\mathcal{C}$. Let $[E] \in \operatorname{Ext}(U, C)$ then

$$
\begin{aligned}
([E]) \operatorname{Ext}(\mu, \psi) \operatorname{Ext}(\theta, \phi) & =[\phi\{(\psi(E \mu)) \theta\}] \\
& =[\phi\{(D(\theta) \psi)((E \mu) \theta)\}], \text { by Proposition } 3.6 \\
& =([E]) \operatorname{Ext}(\theta \mu,(D(\theta) \psi) \phi) \\
& =([E]) \operatorname{Ext}((\theta, \phi)(\mu, \psi)) .
\end{aligned}
$$

Therefore, $\operatorname{Ext}(\mu, \psi) \operatorname{Ext}(\theta, \phi)=\operatorname{Ext}((\theta, \phi)(\mu, \psi))$. Hence $\operatorname{Ext}(-,-)$ is a contravariant functor.

This work is part of the author's Ph.D. thesis (University of Madras, December 1978). The author wishes to express sincere thanks to his supervisor Professor V. K. Balachandran for his encouragement and help throughout preparation of this paper.

\section{References}

G. Lallement (1967), 'Demi-groupes réguliers', Ann. Mat. Pura Appl. 87, 47-130.

H. Lausch (1975), 'Cohomology of inverse semigroups', J. Algebra 35, 273-303.

J. Leech (1975), ' $\mathcal{K}$-coextensions of monoids', Mem. Amer. Math. Soc. 1, no. 157 (Amer. Math. Soc., Providence, R.I.).

M. Loganathan (1981), 'Cohomology of inverse semigroups', to appear.

S. Mac Lane (1963), Homology (Springer-Verlag, New York).

S. Mac Lane (1971), Categories for the working mathematician (Springer-Verlag. New York).

K. S. S. Nambooripad (1979), 'Structure of regular semigroups I', Mem. Amer. Math. Soc. 22, no. 224 (Amer. Math. Soc., Providence, R.I.).

S. Sribala (1977), 'Cohomology and extension of inverse semigroup', J. Algebra 47, 1-17.

Ramanujan Institute of Mathematics

University of Madras

Madras-600 005

India 